\title{
Objective patterning of uroflowmetry curves in children with daytime and nighttime wetting.
}

\section{$\operatorname{AUTHOR}(\mathrm{S}):$}

Kanematsu, Akihiro; Johnin, Kazuyoshi; Yoshimura, Koji; Okubo, Kazutoshi; Aoki, Katsuya; Watanabe, Masato; Yoshino, Kaoru; Tanaka, Shiro; Tanikaze, Saburo; Ogawa, Osamu

\section{CITATION:}

Kanematsu, Akihiro ...[et al]. Objective patterning of uroflowmetry curves in children with daytime and nighttime wetting.. The Journal of urology 2010, 184(4 Supplement 1): 1674 1679

\section{ISSUE DATE:}

2010-10

URL:

http://hdl.handle.net/2433/126740

\section{RIGHT:}

(C) 2010 American Urological Association Education and Research, Inc. Published by Elsevier Inc.; この論文は出版社版でありません。引用の際には出版社版をご確認ご利用 ください。; This is not the published version. Please cite only the published version. 


\section{An objective patterning of uroflowmetry curves in children with daytime and nighttime wetting}

Akihiro Kanematsu $^{1)}$, Kazuyoshi Johnin ${ }^{2)}$, Koji Yoshimura ${ }^{1)}$, Kazutoshi Okubo ${ }^{1)}$,

Katsuya Aoki ${ }^{3)}$, MasatoWatanabe ${ }^{4)}$, Kaoru Yoshino ${ }^{4)}$, Shiro Tanaka ${ }^{5)}$, Saburo

Tanikaze $^{4)}$ and Osamu Ogawa ${ }^{1)}$

1) Department of Urology, Kyoto Universtiy

2) Department of Urology, Shiga University of Medical Science

3) Department of Urology, Nara Medical University

4) Department of Urology, Aichi Children's Health and Medical Center

5) Division of Clinical Trial Design \& Management, Translational Research

Center, Kyoto University Hospital

Running head: Uroflowmetry patterning in children

Key Words: uroflowmetry, enuresis, incontinence, pediatric 


\section{Abstract}

Purpose: Interpretation of pediatric UFM curves is incompletely standardized. Therefore, we propose a new objective patterning.

Materials and Methods: UFM curves were obtained from 100 children presenting with daytime incontinence or enuresis. Each curve was compared with a standard curve generated from a published nomogram, and a new patterning method was formulated. First, Staccato and Interrupted patterns were defined using International Children's Continence Society (ICCS) criteria. Next, the remaining curves were divided by the deviation of maximal flow rate (MFR) from the median value of the nomogram as Tower ( $>130 \%)$, 'Not abnormal' $(70-130 \%)$, and Plateau $(<70 \%)$ patterns. The correlation between the presenting symptom and the patterns or other UFM parameters was evaluated. Finally, six pediatric urologists also patterned the same curves subjectively.

Results: All of the curves could be classified to one of the defined patterns using this method. The distribution of the patterns reflected the spectrum of presenting symptoms, with more Tower, Interrupted, and Staccato patterns in children with daytime wetting than in those with monosymptomatic enuresis. Age-adjusted voided volume was also smaller in the former group, but post-void residual, MFR and average flow rate were not correlated with presenting symptoms. Subjective patterning showed marked inter-observer differences. When the patterning applied by the present method was used as a reference, the sensitivity of the observers for abnormal patterns was inversely correlated with their specificity. Conclusion: Subjective UFM patterning is liable to personal bias. The proposed method enables objective patterning that complies with ICCS standardization and 
clinical presentation. 


\section{Introduction}

Uroflowmetry (UFM) is the most common diagnostic urodynamic procedure for evaluating children with lower urinary tract problems. ${ }^{1,2}$ In adults and in children, it is useful for assessing bladder outlet obstruction, and the maximal flow rate (MFR) is a reliable parameter for that purpose. In addition, UFM is widely used for diagnosing children with daytime and nighttime wetting, which are highly prevalent conditions during childhood. ${ }^{3-6}$ It has been postulated that UFM can detect underlying bladder problems by defining certain abnormal urination patterns. The International Children's Continence Society (ICCS) proposed standardizing the terminology for such abnormal curves, and classified them into Bell (for normal), Tower, Plateau, Staccato and Interrupted patterns. ${ }^{1}$ However, the patterning of UFM curves is incompletely standardized and is largely at the physician's subjective discretion. In short, UFM is a first-line, non-invasive test to assess voiding function, but the interpretation of the results is not scientifically standardized for evaluating pediatric bladder problems, and some authors question its utility.

Here, we attempted to develop an objective patterning method, starting by comparing clinical UFM curves with corresponding standard curves. The method was validated by reference to clinical symptoms and compared with subjective patterning performed by six pediatric urologists. 
Materials and Methods

Clinical data sets: UFM curves were obtained from 100 consecutive children aged

6-15 years old who presented at Shiga Medical Center for Children for daytime incontinence and/or enuresis. Patients with spinal lesions, major urinary tract anomalies detectable by screening ultrasound or urinary tract infection were excluded.

There were 41 children with daytime incontinence with or without enuresis (Group A), 28 with non-monosymptomatic enuresis (non-MNE) without daytime incontinence (i.e. children with nocturnal enuresis with daytime symptoms like urgency, frequnecy, or holding but no episode of daytime wetting, Group B), and 31 with monosymptomatic enuresis (MNE, Group C). All tests were performed at full bladder, on their first office visit.

The age-adjusted capacity of the children was calculated using published formula for Japanese children, $($ capacity $)=($ age +2$) \times 25 .{ }^{9}$ Residual urine volume was calculated from ultrasound images using the formula $\mathrm{Q}=\mathrm{abc} / 2$.

Formulation of the patterning method (Figure 1). Step 1: Generation of tentative standard curves: Tentative standard uroflowmetry curves were generated with a shape similar to our unpublished data in healthy adults, and fitting to average and maximal flow rate for corresponding voided volume in a published pediatric nomogram. ${ }^{10,11}$ Step 2: All of the clinical curves were scanned and digitized by GraphCel, a free on-line software (http://t_kobo.at.infoseek.co.jp/grp/), for further analysis by Microsoft Excel (Microsoft, Redmond, WA). The standard curves were also transformed to Excel files. Step 3: Each curve was directly compared with the corresponding standard curve by overlapping, and deviation from the standard curve was considered abnormal. 
As a result, the following patterning method was formulated. First, Staccato and Interrupted patterns were defined by the ICCS criteria. ${ }^{1}$ We grouped them together as one group in further analysis, as differentiation between the two patterns was considered to be difficult owing to the indefinite duration of interruption. Next, the other curves were classified by deviation of the MFR from the median value of the nomogram as Tower, 'Not abnormal', and Plateau patterns.

Validation of the patterning method. First, each clinical group was stratified by standard parameters, i.e. voided volume, MFR, average flow rate (AFR), and postvoid residual (PVR). Second, each group was stratified by the new patterns, setting cutoff lines of MFR for defining Tower and Plateau patterns. Finally, six pediatric urologists subjectively patterned the same curves. The urologists were informed about age and sex but not about the clinical presentation of the patients. Assuming the patterning obtained with the present method as a reference, we assessed agreement of the subjective and objective patterning by Kappa coefficients and 95\% confidence intervals for each pair of six raters and the objective patterning. Statistical analysis was performed with Statview software (Abacus, Burlington, MA) and SAS software (SAS, Cary, NC). P values $<0.05$ were considered as significant.

Results

Demographic characteristics. The demographic characteristics of the 100 children undergoing UFM are shown in Table 1. There was no statistical difference in the sex ratio, but the age distribution was significantly shifted to a younger age in Group A $(\mathrm{p}<0.05)$

Relationship between standard parameters of uroflowmetry and presenting symptoms (Figure 2). The age-adjusted voided volume was smaller in children with 
daytime incontinence than those without. PVR, MFR and AFR, as a single parameter, were not correlated with the presenting symptoms.

Relationship between the new uroflowmetry patterning and the presenting symptoms (Figure 3). Using the present method, all of the curves could be classified to one of the patterns. We have tentatively set multiple cutoff lines by changing it from 5070\%, and 130-150\% respectively. Finally, we set the cutoff values of MFR for defining Tower and Plateau patterns at 70 and $130 \%$, since it seemed to represent average result of the raters, and the distribution of the patterns reflected the spectrum of presenting symptoms, with more Tower, Interrupted, and Staccato patterns for daytime incontinence and non-MNE, and less in those with MNE (Figure $3)$.

Comparison of subjective and objective patterning (Figure 4). The six examiners who performed subjective patterning judged that $4-14 \%$ (mean, $10.5 \%$ ) of the curves were 'unclassifiable'. The judgment for the remaining curves showed marked interobserver differences. There was a wide range of inter-observer difference, from 0.21-0.64. The range of agreement rate between the raters and the objective patterning was narrower, from 0.37-0.55 (Table 2). When the patterning applied using the present method was set as the reference, the sensitivity among the observers for abnormal patterns was inversely correlated with their specificity (Figure 4). In 30 cases, 3 raters or more made the same diagnosis, discrepant from the objective patterning. Majority of these, $22 / 30(73.3 \%)$ were related with discrimination of Tower and Plateau pattern from normal pattern. (three examples are shown in Figure 5).

Discussion 
This paper presents a new objective patterning method complying with the ICCS standardization and reflecting clinical symptoms.

In this report, we showed that MFR and AFR, standard parameters of UFM, do not reflect the clinical symptoms by themselves (Figure 2). The only factor reflecting the symptom was age-adjusted voided volume, which should be better represented by a flow-void chart. The poor utility of MFR and AFR is because of the presence of Staccato and Interrupted patterns in many children. Although these patterns manifest the worst voiding pattern, fractionated peaks and relatively long voiding time mean they are classified at an average level of MFR and AFR. Initial definition of these patterns leaves single-peaked curves, which are more manageable by a conventional parameter, namely MFR. Indeed, MFR, which is considered to be 'the most relevant variable' according to the ICCS standardization committee, ${ }^{1}$ can be fully utilized to differentiate Tower and Plateau patterns from normal uroflow curves.

The first point of the present study is that UFM patterns alone, in the absence of clinical information, may reflect the clinical symptoms of children with daytime and nighttime wetting (Figure 3). Daytime incontinence, which is considered to be a manifestation of overactive bladder or dysfunctional voiding, is more associated with Tower, Staccato or Interrupted patterns than MNE. Although such features have been postulated as the rationale to perform UFM as first-line examination for children with incontinence and enuresis, the reliability of UFM has been questioned because of the lack of an objective standard. ${ }^{7,8}$ Our findings may reconcile the present consensus of the clinical utility of UFM and the concurrent skepticism against it.

The second point is that subjective patterning is liable to personal bias (Table 2). When the patterning using the present method was used as a reference, the sensitivity of the observers for abnormal patterns was inversely correlated with their specificity 
(Figure 4). This suggests that each physician may have internally consistent cutoffs for patterning, which may not be consistent with that of other physicians. Without standardization of the evaluation method, we can neither discuss usefulness or uselessness of UFM for diagnosing and treating pediatric overactive bladder or dysfunctional voiding, nor can we compare UFM data with other parameters such as bladder wall thickness or external sphincter electromyography.

In this study, we deliberately avoided using the term 'Bell' to define the normal pattern because the term 'Bell' may frequently result in over-interpretation of subtle changes in the curves. For example, a curve nearly identical to the standard curve could be interpreted as a Plateau or Tower (Figure 5B and 5C). Referring to a standard nomogram is sufficient to avoid such confusion.

There is one remaining question regarding this study, which is the lack of normal control. The cutoff line for MFR in the present patterning method was defined rather arbitrarily, to translate the physicians' judgments to a mathematical standard. The proposed cutoff may differentiate MNE from daytime incontinence and non-MNE, but it is unclear whether it can differentiate between dry and wet children. Hence, we cannot conclude from the present result whether the $38.7 \%$ 'abnormal' patterns found in MNE patients are higher or identical with that of completely dry children. This point is particularly important because it is still unclear whether pure MNE children have normal bladder function or not, as enuretic children do exhibit bladder overactivity at night, causing cortical arousal. ${ }^{12,13}$ Further validation of the present results on an epidemiological basis would further elucidate the true pathophysiology of enuretic children, and the usefulness of UFM for their evaluation. 


\section{Conclusions}

Here, we formulated an objective patterning of UFM, complying with ICCS standardization and clinical presentation of enuresis. This patterning may replace the current subjective UFM patterning, which is liable to personal bias. Further validation of this new patterning is needed with reference to a population-based control. 


\section{References}

1. Neveus, T., von Gontard, A., Hoebeke, P. et al.: The standardization of terminology of lower urinary tract function in children and adolescents: report from the Standardization Committee of the International Children's Continence Society. J Urol, 176: 314, 2006

2. Neveus, T.: The new International Children's Continence Society's terminology for the pediatric lower urinary tract--why it has been set up and why we should use it. Pediatr Nephrol, 23: 1931, 2008

3. Sureshkumar, P., Jones, M., Cumming, R. et al.: A population based study of 2,856 school-age children with urinary incontinence. J Urol, 181: 808, 2009

4. Chung, J. M., Lee, S. D., Kang, D. I. et al.: Prevalence and associated factors of overactive bladder in Korean children 5-13 years old: a nationwide multicenter study. Urology, 73: 63, 2009

5. Kajiwara, M., Inoue, K., Usui, A. et al.: The micturition habits and prevalence of daytime urinary incontinence in Japanese primary school children. J Urol, 171: 403, 2004

6. Kajiwara, M., Inoue, K., Kato, M. et al.: Nocturnal enuresis and overactive bladder in children: an epidemiological study. Int J Urol, 13: 36, 2006

7. Jensen, K. M., Nielsen, K. K., Kristensen, E. S. et al.: Uroflowmetry in neurologically normal children with voiding disorders. Scand J Urol Nephrol, 19: 81,1985

8. Dogan, H. S., Akpinar, B., Gurocak, S. et al.: Non-invasive evaluation of voiding function in asymptomatic primary school children. Pediatr Nephrol, 23: 1115,2008 
9. Hamano, S., Yamanishi, T., Igarashi, T. et al.: Evaluation of functional bladder capacity in Japanese children. Int J Urol, 6: 226, 1999

10. Kajbafzadeh, A. M., Yazdi, C. A., Rouhi, O. et al.: Uroflowmetry nomogram in Iranian children aged 7 to 14 years. BMC Urol, 5: 3, 2005

11. Okubo, K., Yoshimura, Kanematsu, A., Nishiyama, H., Ogawa, O.: Average shapes of uroflow curves of adult men. Abstract presented in Annual Meeting of International Continence Society, 2009

12. Yeung, C. K., Diao, M., Sreedhar, B.: Cortical arousal in children with severe enuresis. N Engl J Med, 358: 2414, 2008

13. Hjalmas, K., Arnold, T., Bower, W. et al.: Nocturnal enuresis: an international evidence based management strategy. J Urol, 171: 2545, 2004 
Figure Legends

Figure 1. Fitting with standard curves. The examined lines are shown in black and the corresponding standard curves are shown in grey. Typical 'Not abnormal' (A), 'Interrupted' or 'Staccato' (B), 'Plateau' (C) and 'Tower' (D) patterns are shown.

Figure 2. Relationship between presenting symptoms and standard UFM parameters

A. Voided volume, expressed as the percentage to age-adjusted standard capacity $(\mathrm{p}<0.01)$.

B. Post-void residual volume.

C. Maximal flow rate (MFR), as stratified by the reference nomogram.

D. Average flow rate (AFR), as stratified by the reference nomogram.

Figure 3.

Stratification of voiding patterns according to the presenting symptoms. More Tower, Staccato, and Interrupted patterns were found in Group A compared with Group C.

Figure 4.

Subjective patterning was compared with the new patterning as a reference. A negative relationship was found between the sensitivity for abnormal patterns (the rate at which a rater judge 'abnormal' curve by the present patterning, as abnormal) and the specificity for abnormal patterns (the rate at which a rater judge 'normal' curve by the present patterning, as normal).

Figure 5. 
In some cases, collective discrepancy was found between the objective and subjective patternings. Standard curves are superimposed and shown as grey lines (bottom).

A. The curve was judged as 'Bell' pattern by all examiners, but classified as 'Plateau' with the objective patterning.

B. The curve was judged as 'Tower' by all examiners, but classified as 'Not abnormal' with the objective patterning.

C. The curve was judged as 'Plateau' by 3 examiners, but classified as 'Not abnormal' with the objective patterning. 
Abbreviations in the Paper

UFM: uroflowmetry

ICCS: International Children's Continence Society

MFR: Maximal Flow Rate

AFR: Average Flow Rate

PVR: Post-void residual urine

MNE: Monosymptomatic nocturnal enuresis 
Table 1. Demographic data of the patients.

\begin{tabular}{|c|c|c|c|c|c|c|c|c|c|c|c|c|}
\hline \multirow{3}{*}{$\begin{array}{l}\text { Average Age } \\
\text { Standard deviation }(\mathrm{m})\end{array}$} & \multicolumn{2}{|c|}{$\begin{array}{r}\text { Group A } \\
\text { Male Female }\end{array}$} & Total & \multicolumn{2}{|c|}{$\begin{array}{l}\text { Group B } \\
\text { Male Female }\end{array}$} & \multirow{2}{*}{$\frac{\text { Total }}{9 \mathrm{y} 0 \mathrm{~m}}$} & \multicolumn{3}{|c|}{$\begin{array}{c}\text { Group C } \\
\text { Male Female Total }\end{array}$} & \multicolumn{2}{|c|}{$\begin{array}{c}\text { Total } \\
\text { Male Female }\end{array}$} & Total \\
\hline & $8 y 4 m$ & $7 y 8 m$ & $8 \mathrm{y} 1 \mathrm{~m} *$ & $8 \mathrm{y} 11$ & $1 \mathrm{~m} 9 \mathrm{y} 4 \mathrm{~m}$ & & $9 \mathrm{y} 0 \mathrm{~m}$ & $9 \mathrm{y} 7 \mathrm{~m}$ & $9 y 2 m$ & $8 \mathrm{y} 7 \mathrm{~m}$ & $8 y 9 m$ & $8 y 8 m$ \\
\hline & 30.6 & 16.9 & 26.8 & 22.7 & 32.1 & 24.4 & 19.3 & 26 & 21 & 25.0 & 25.0 & 24.9 \\
\hline \multicolumn{13}{|l|}{ Age group } \\
\hline $6-7$ & 15 & 8 & 23 & 8 & 2 & 10 & 6 & 1 & 7 & 29 & 11 & 40 \\
\hline $8-9$ & 8 & 5 & 13 & 7 & 3 & 10 & 12 & 3 & 15 & 27 & 11 & 38 \\
\hline $10-11$ & 2 & 1 & 3 & 7 & 0 & 7 & 4 & 3 & 7 & 13 & 4 & 17 \\
\hline $12-13$ & 0 & 0 & 0 & 0 & 0 & 0 & 1 & 1 & 2 & 1 & 1 & 2 \\
\hline $14-15$ & 2 & 0 & 2 & 0 & 1 & 1 & 0 & 0 & 0 & 2 & 1 & 3 \\
\hline Total Number & 27 & 14 & 41 & 22 & 6 & 28 & 23 & 8 & 31 & 72 & 28 & 100 \\
\hline
\end{tabular}

*: Younger than other groups $(\mathrm{p}<0.05)$. 
Table 2. Kappa coefficients for each pair of six raters and the objective patterning

\begin{tabular}{|c|c|c|c|c|c|c|c|c|c|c|c|c|}
\hline \multirow[t]{2}{*}{ Rater } & \multicolumn{3}{|c|}{1} & \multicolumn{3}{|c|}{2} & \multicolumn{3}{|c|}{3} & \multicolumn{3}{|c|}{4} \\
\hline & \multicolumn{3}{|c|}{ Kappa $95 \%$ CI } & \multicolumn{3}{|c|}{ Kappa $95 \%$ CI } & \multicolumn{3}{|c|}{ Kappa $95 \%$ CI } & \multicolumn{3}{|c|}{ Kappa 95\% CI } \\
\hline 1 & - & - & - & - & - & - & - & - & - & - & - & - \\
\hline 2 & 0.64 & 0.47 & 0.82 & - & - & - & - & - & - & - & - & - \\
\hline 3 & 0.36 & 0.19 & 0.53 & 0.42 & 0.27 & 0.57 & - & - & - & - & - & - \\
\hline 4 & 0.28 & 0.10 & 0.47 & 0.38 & 0.22 & 0.54 & 0.49 & 0.36 & 0.63 & - & - & - \\
\hline 5 & 0.21 & -0.01 & 0.42 & 0.26 & 0.07 & 0.45 & 0.49 & 0.36 & 0.63 & 0.38 & 0.23 & 0.53 \\
\hline 6 & 0.37 & 0.19 & 0.55 & 0.44 & 0.28 & 0.59 & 0.61 & 0.48 & 0.74 & 0.60 & 0.46 & 0.73 \\
\hline Objective & & & & & & & & & & & & \\
\hline Patterning & 0.37 & 0.18 & 0.55 & 0.55 & 0.40 & 0.71 & 0.43 & 0.29 & 0.57 & 0.43 & 0.29 & 0.57 \\
\hline
\end{tabular}

\begin{tabular}{cllllll}
\hline Rater & \multicolumn{2}{c}{5} & \multicolumn{2}{c}{6} \\
\hline & \multicolumn{2}{c}{ Kappa $95 \%$ CI } & \multicolumn{2}{c}{ Kappa 95\% CI } \\
\hline 1 & - & - & - & - & - & - \\
2 & - & - & - & - & - & - \\
3 & - & - & - & - & - & - \\
4 & - & - & - & - & - & - \\
5 & - & - & - & - & - & - \\
6 & 0.55 & 0.41 & 0.69 & - & - & - \\
Objective & & & & & & \\
Patterning & 0.42 & 0.27 & 0.57 & 0.45 & 0.30 & 0.60 \\
\hline
\end{tabular}


A Normal

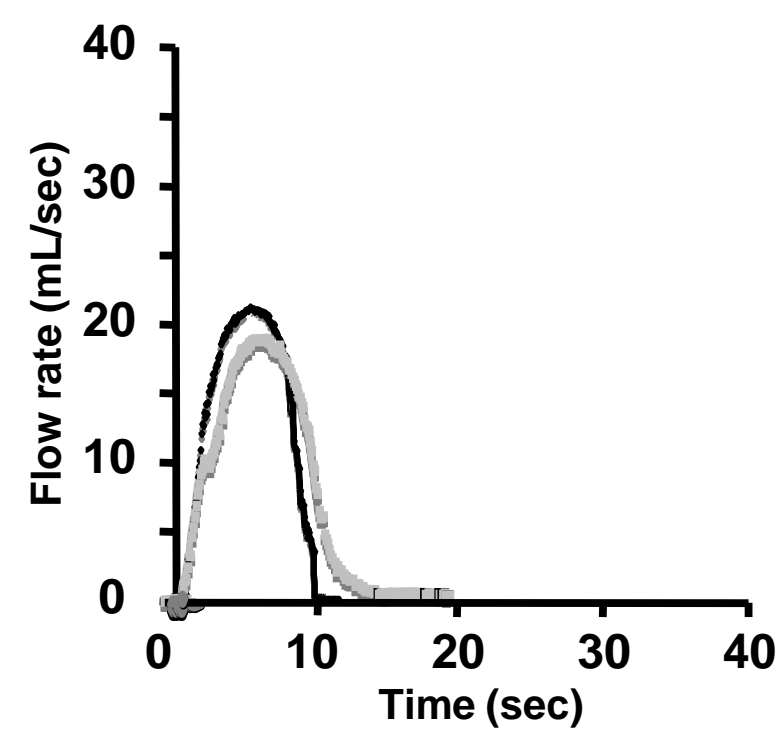

C

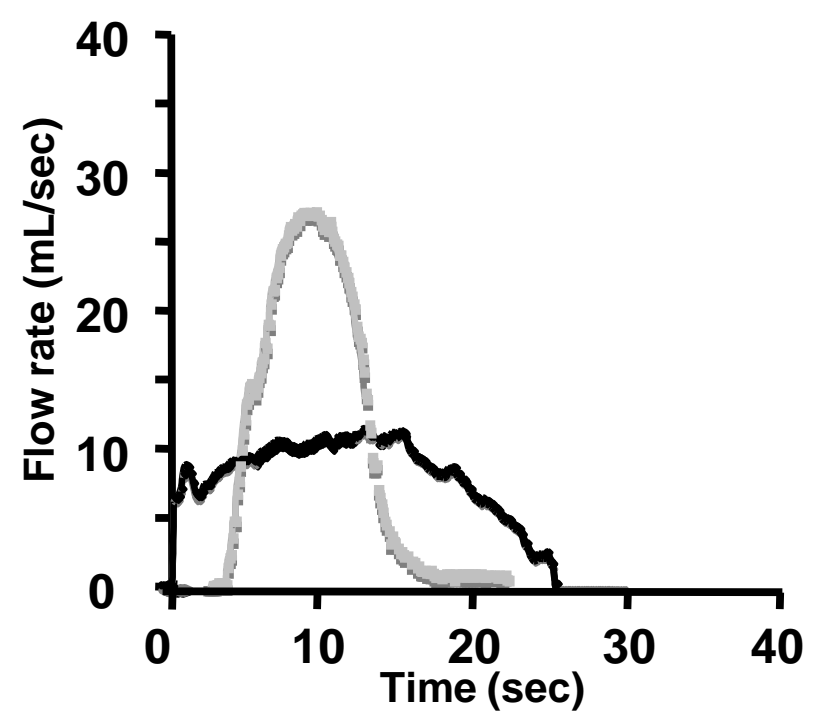

B Staccato

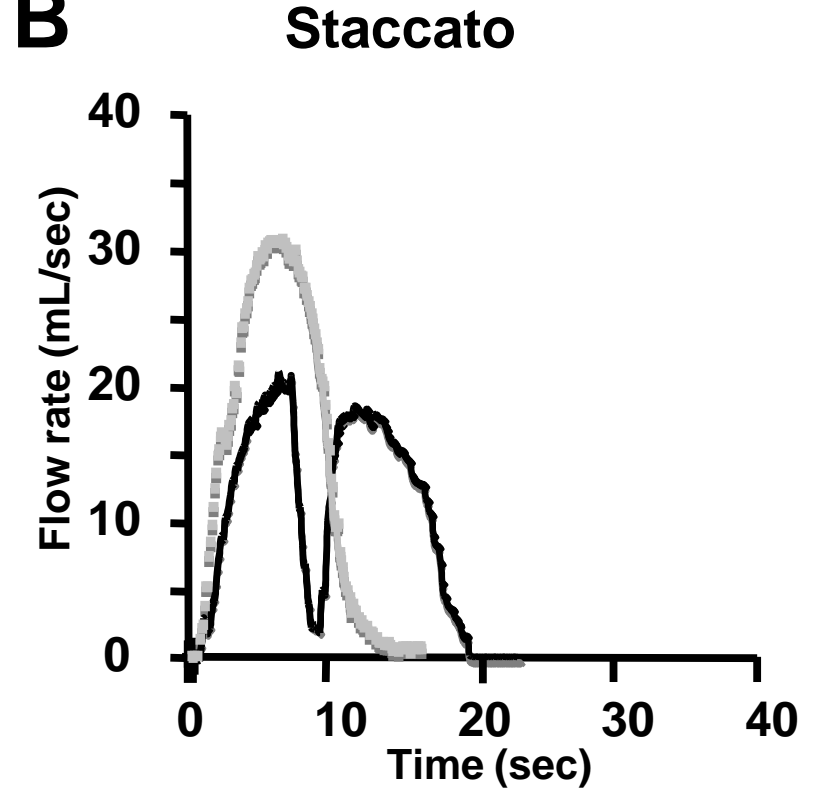

D Tower

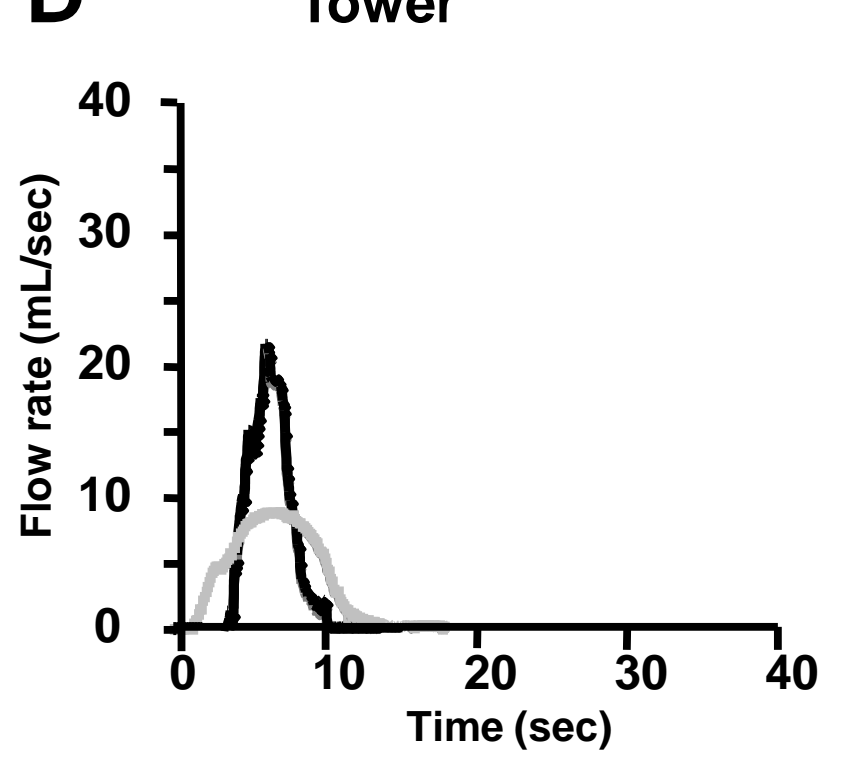


A

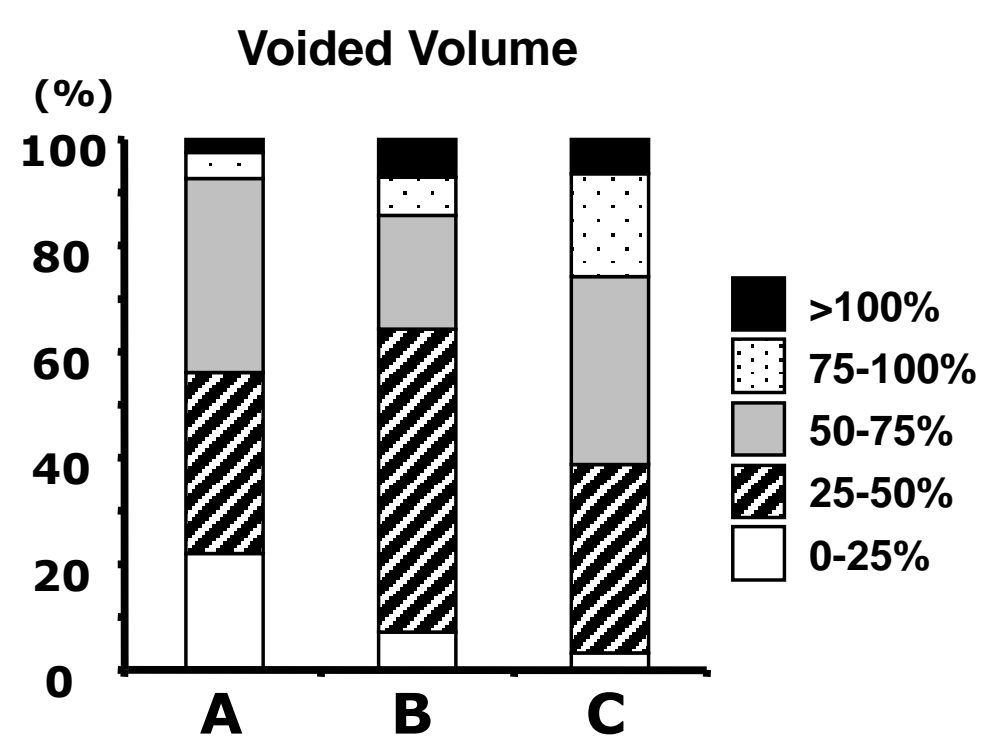

C

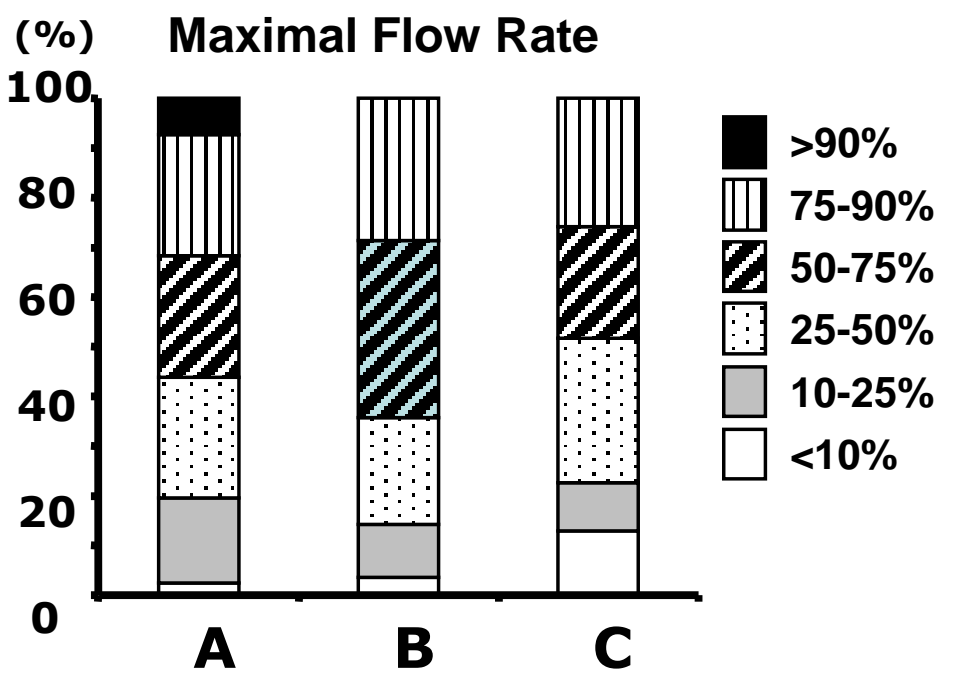

B

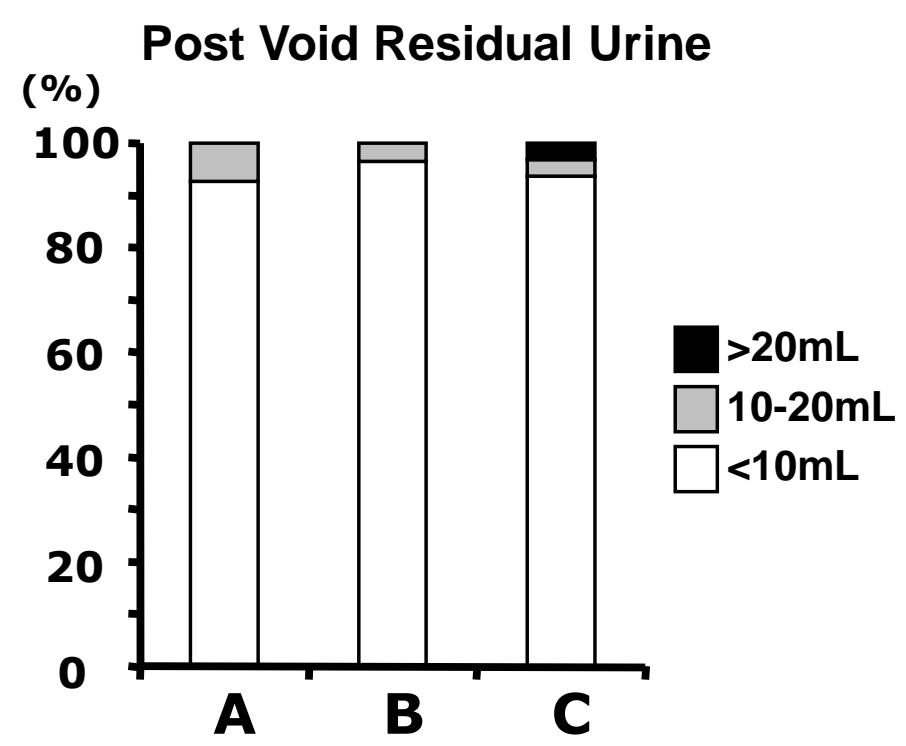

D

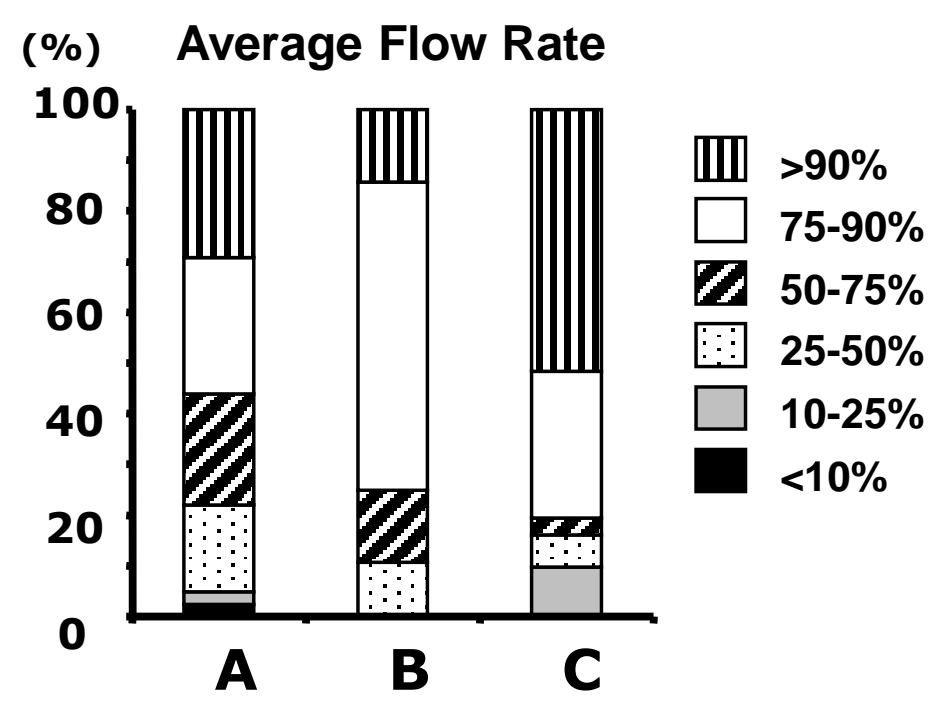


(\%)

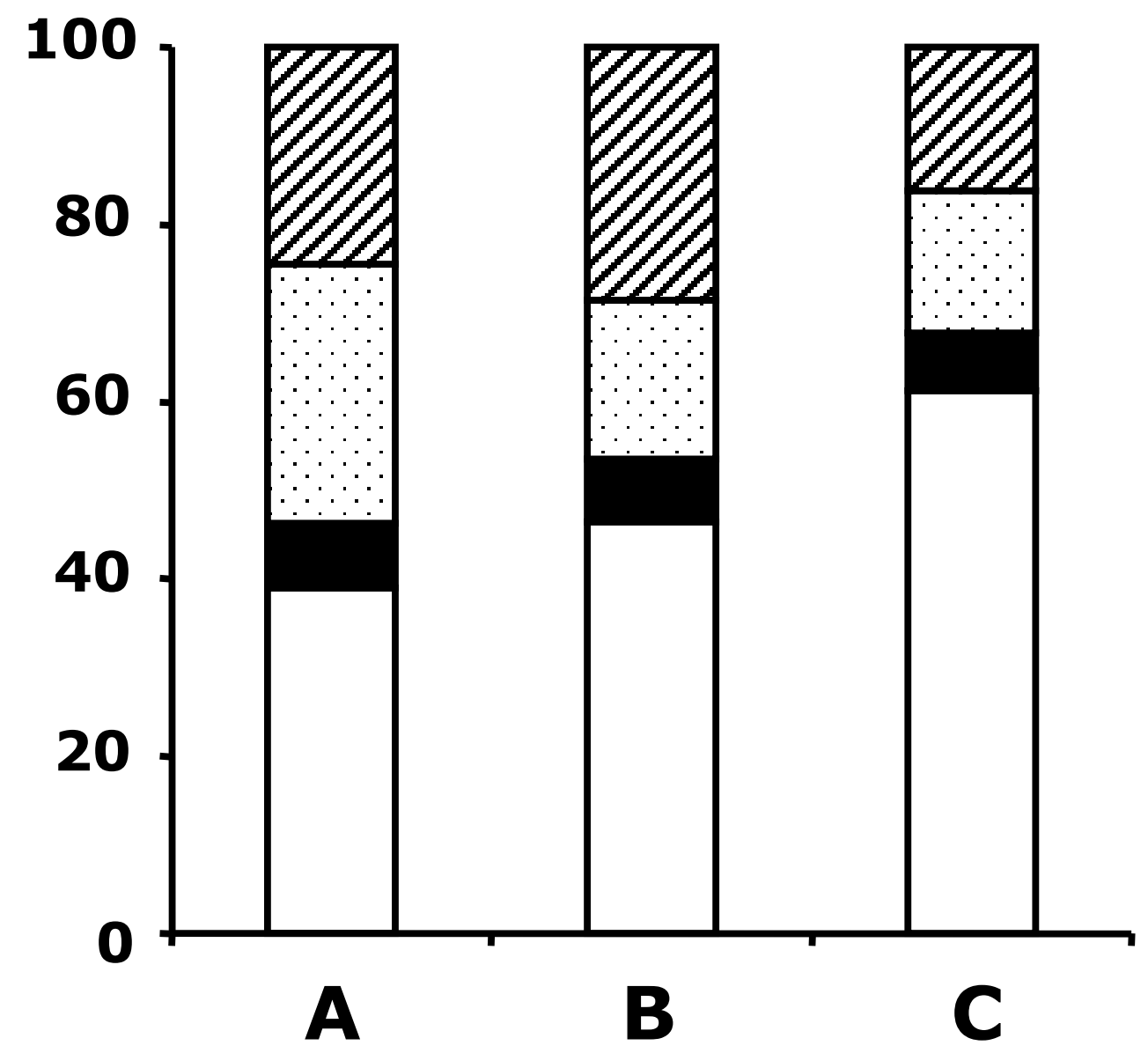

$\square$ Tower

Staccato \&

Interrupted

Dlateau

$\square \quad$ Not abnormal 


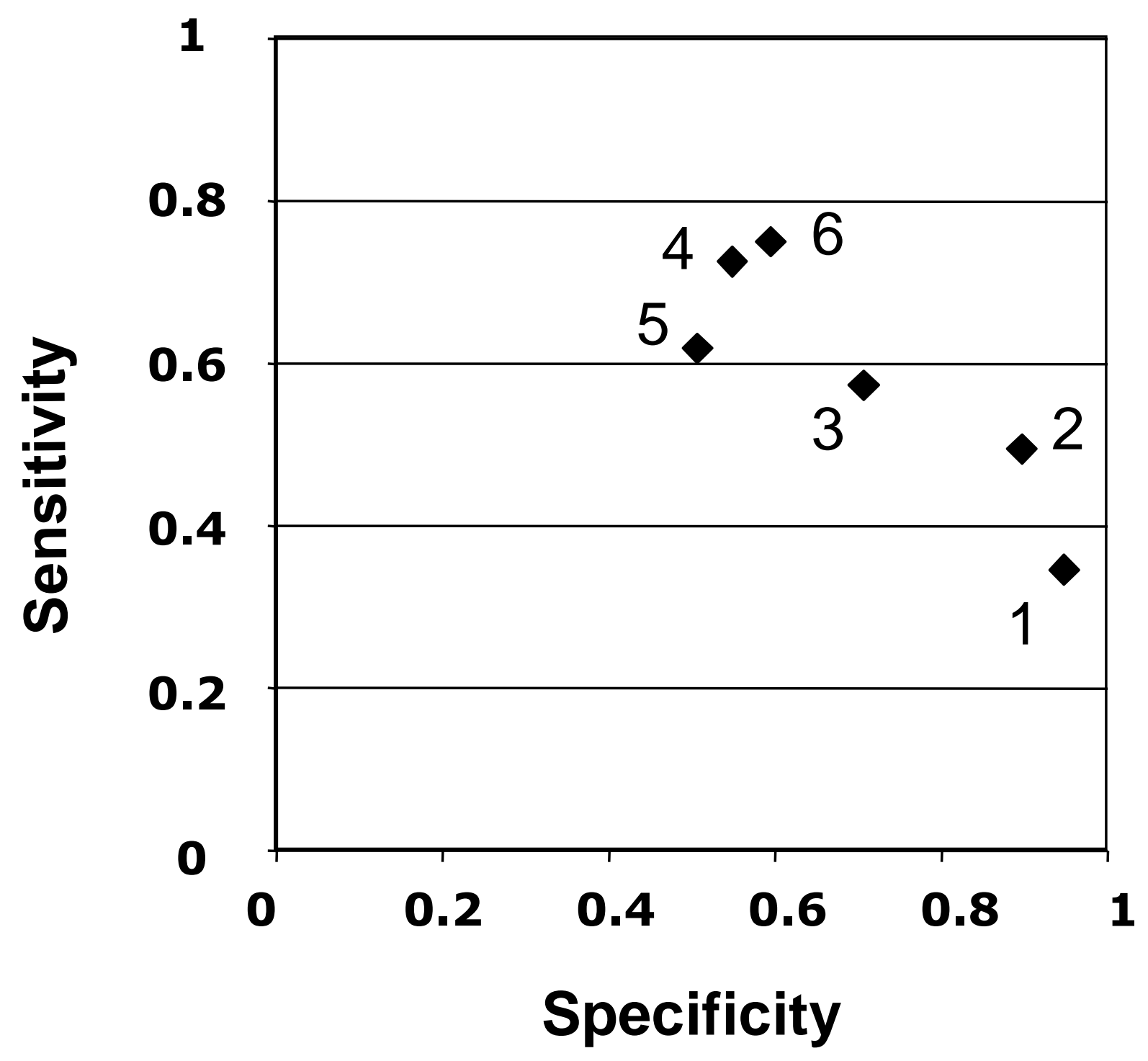


A All judged as 'Bell'
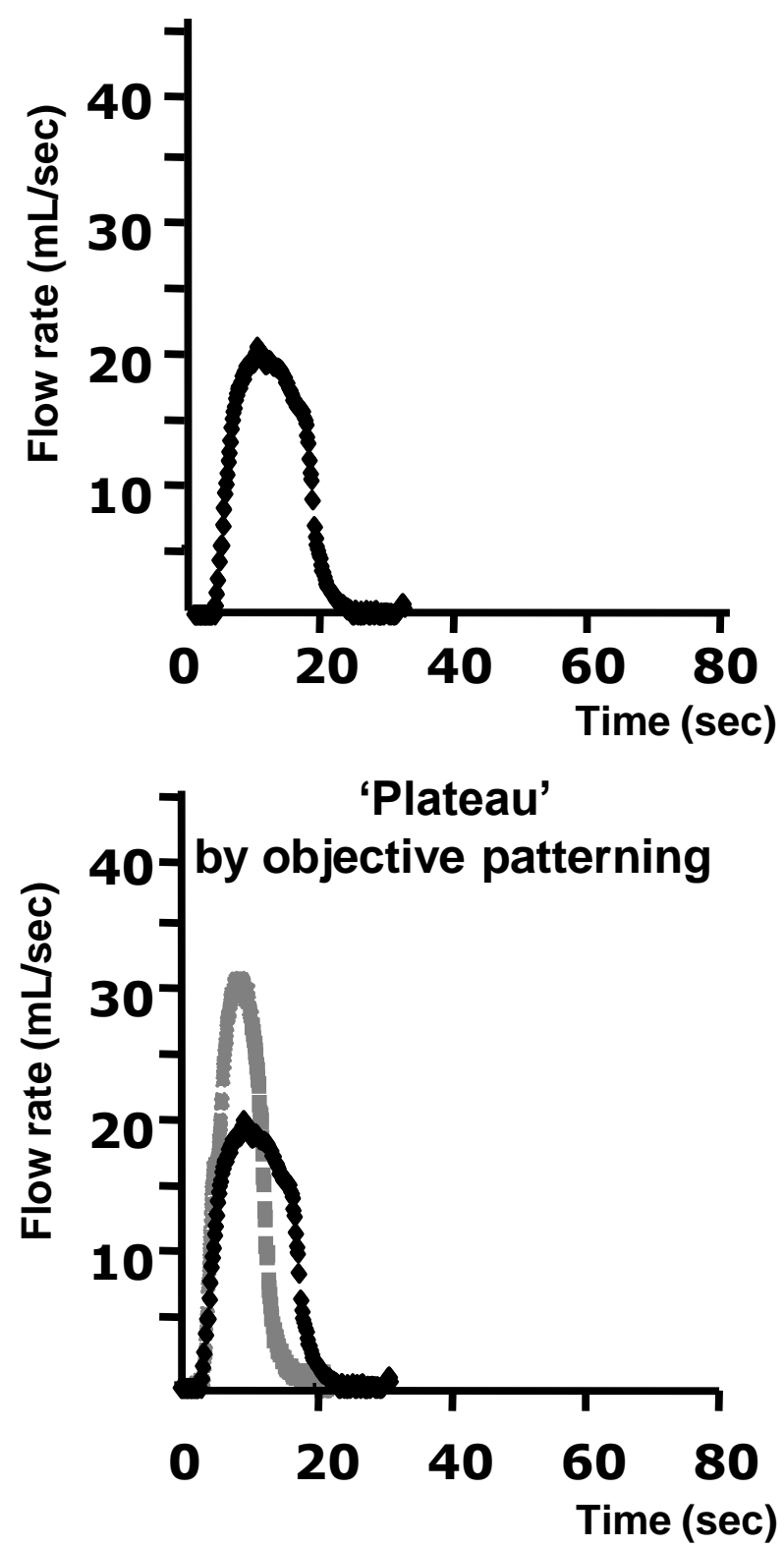

B All judged as 'Tower'
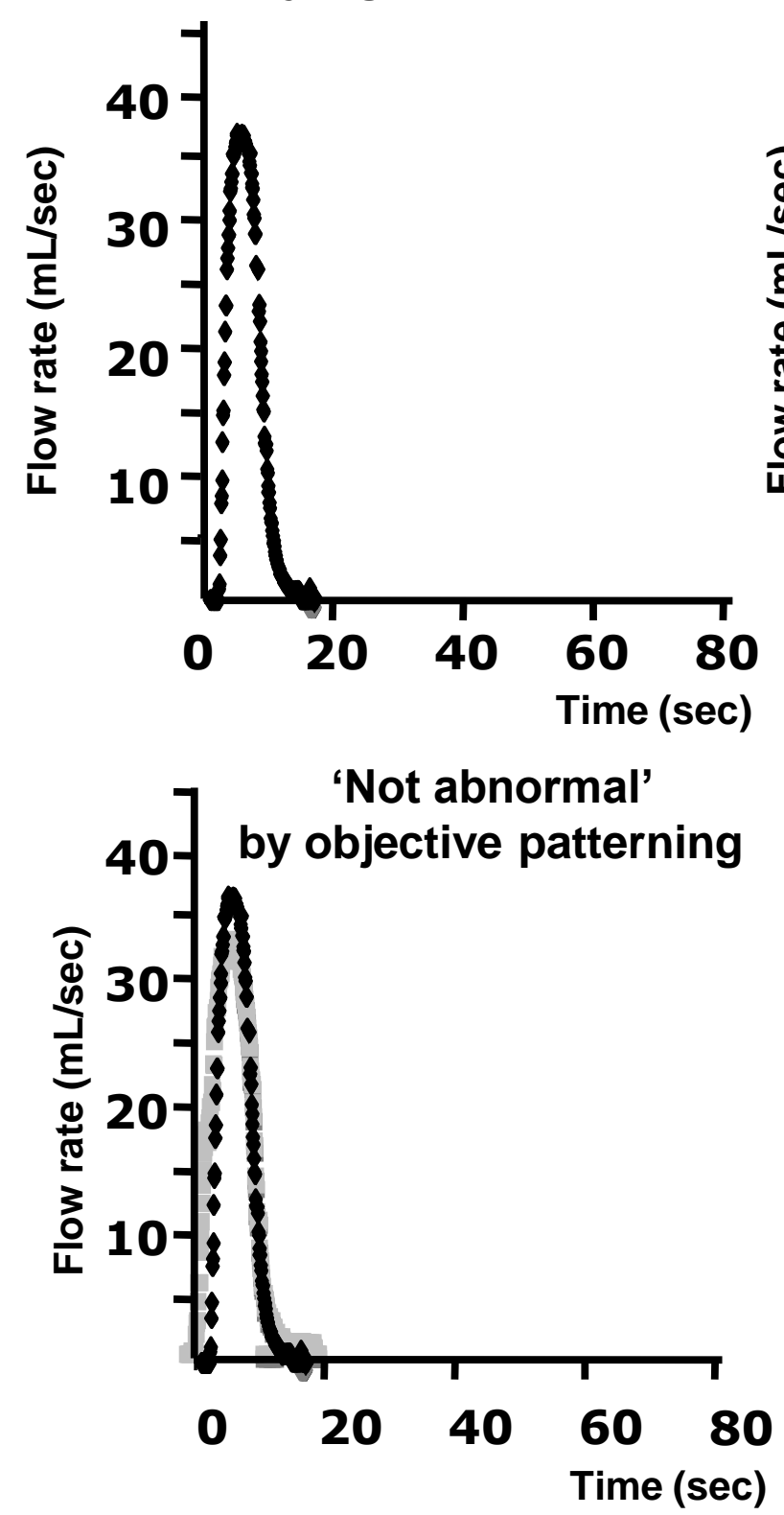

C Three judged as 'Plateau'
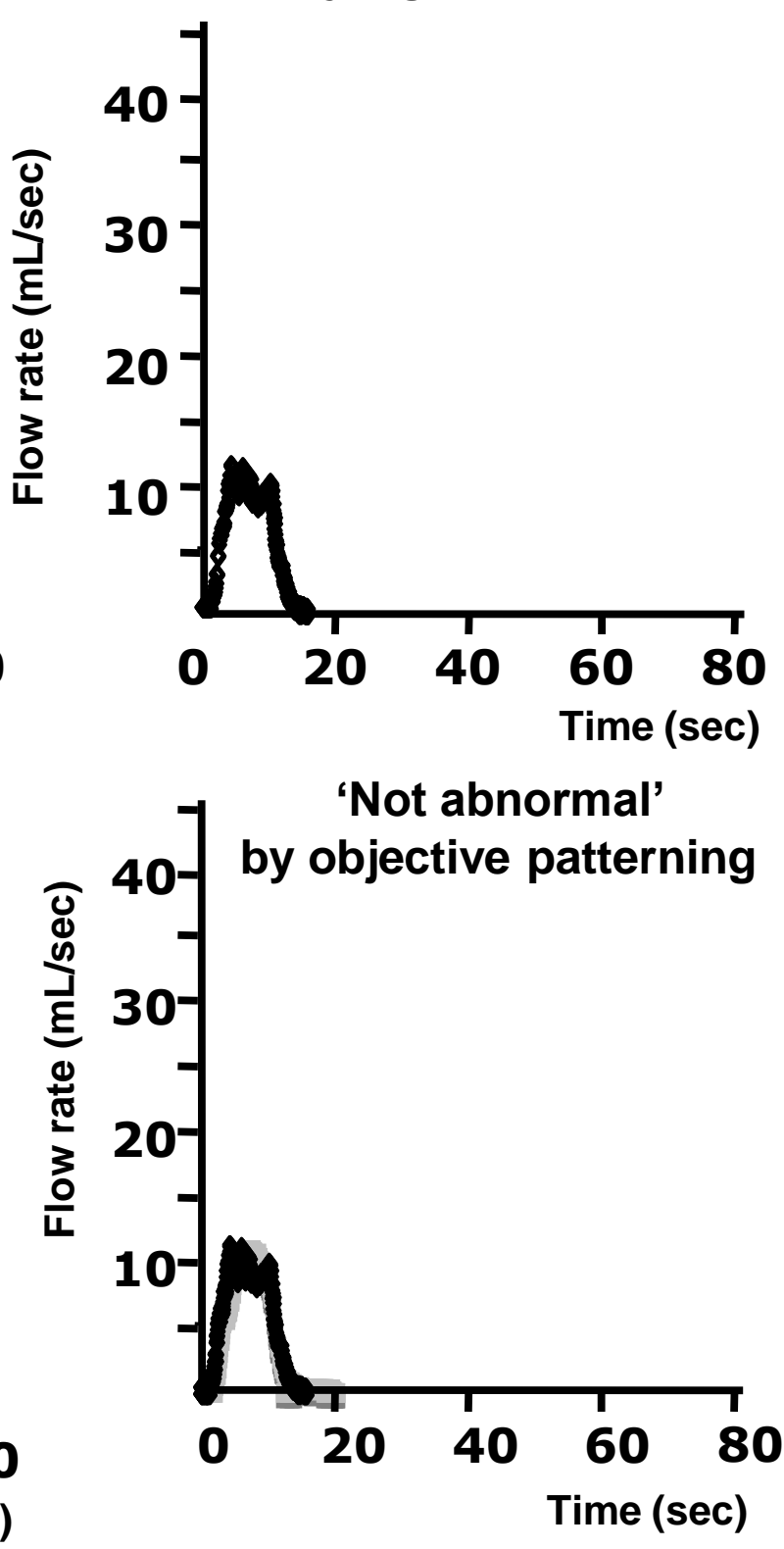